\title{
Evaluation of the retinal, choroidal, and nerve fiber layer thickness changes in patients with toxic anterior segment syndrome
}

\author{
Nir Sorkin • Dafna Goldenberg • Amir Rosenblatt • \\ Gabi Shemesh
}

Received: 9 August 2013 / Revised: 4 November 2014 / Accepted: 20 November 2014 / Published online: 3 December 2014

(C) The Author(s) 2014. This article is published with open access at Springerlink.com

\begin{abstract}
Purpose To evaluate changes in choroidal, retinal, and nerve fiber layer (NFL) thickness following toxic anterior segment syndrome (TASS).

Methods Macular and peripapillary choroidal thickness was measured using enhanced depth imaging (EDI) optical coherence tomography (OCT) on the day of the diagnosis and on three follow-up exams (months 1 to 4). A similar OCT analysis of central retinal and NFL thickness was performed.

Results Thirteen TASS patients were included. Average age was $72.8 \pm 8.7$ years. Macular choroidal thickness in the superior, subfoveal, and nasal macula in the study eye was larger than the control eye and decreased at months $2-4$. This was statistically significant only for the superior macula $(\mathrm{p}=0.004)$. Peripapillary choroidal thickness was larger in the study eye at baseline compared with the control eye - significantly so in the nasal $(p=0.026)$ and inferior $(p=0.033)$ locations. Peripapillary choroidal thickness peaked at the baseline or 1st month exam and decreased thereafter. Retinal thickness increased significantly with time, peaking at the 2 nd month and decreasing thereafter. No changes were found in the NFL. Conclusions TASS may have a transient effect on the choroid. Changes in retinal thickness are probably a normal transient postoperative response and not a result of TASS.
\end{abstract}

Keywords Choroid · Enhanced depth imaging · Optical coherence tomography $\cdot$ Retina $\cdot$ Toxic anterior segment syndrome

This paper was presented at the 2013 ASCRS Symposium and Congress, San Francisco, USA (April, 2013)

N. Sorkin $(\square) \cdot$ D. Goldenberg $\cdot$ A. Rosenblatt $\cdot$ G. Shemesh Department of Ophthalmology, Tel Aviv Medical Center and the Sackler Faculty of Medicine, Tel Aviv University,

6 Weizmann Street, Tel Aviv 6423906, Israel

e-mail: nir_sorkin@yahoo.com

\section{Introduction}

Cataract surgery is one of the most common surgeries performed worldwide, with a high rate of success. Toxic anterior segment syndrome (TASS), a term coined by Monson et al. in 1992 [1], is a postoperative complication reported in increasing numbers. It is a sterile, postoperative inflammation occurring early after anterior segment surgery, generally appearing within $12-48 \mathrm{~h}$ [2]. It may result in damage to various intraocular tissues.

Typically, TASS presents acutely, but rarely can have a delayed onset. Symptoms include blurry vision, discomfort, and minimal pain. Signs include eye redness, fibrin formation with or without hypopyon, inflammatory membranes, and diffuse corneal edema secondary to endothelial damage. In addition, potential damage to the iris and trabecular meshwork may occur. The inflammation is limited to the anterior segment. TASS can present sporadically or in outbreaks [3], possible causes are numerous [4], but in many of the cases the cause is not identified. The outcome of topical steroidal drops and close monitoring in the management of TASS is excellent [5]. However, severe complications, such as irreversible visual loss, permanent endothelial damage, corneal decompensation, a permanently dilated pupil, or glaucoma due to permanent damage to the trabecular meshwork, may occur $[2,6]$.

TASS has many similarities to other postoperative inflammatory processes, such as uveitis and endophthalmitis $[7,8]$. However, it can be distinguished from endophthalmitis as it seems to be limited to the anterior segment [2].

In the past, choroidal thickness could not be accurately measured. Recent advancements in optical coherence tomography (OCT) with the use of enhanced depth imaging (EDI) allow more accurate evaluation of choroidal thickness in a reproducible manner, as shown in previous studies [9-11]. In the field of inflammatory eye diseases, this technique has been 
used to demonstrate changes in choroidal thickness in VogtKoyanagi-Harada panuveitis subjects [12]. The inflammatory process seen in TASS influences anterior uveal structures. Our aim was to investigate whether these inflammatory changes have an effect on the posterior uvea and retina and whether subtle choroidal and retinal changes, which cannot be noticed clinically, exist.

\section{Materials and methods}

This prospective study adhered to the tenets of the Declaration of Helsinki and was approved by the institutional review board (IRB) of the Tel Aviv Medical Center. Informed consent was obtained from all patients.

\section{Participants}

Thirteen eyes of 13 subjects, consecutively diagnosed with TASS following cataract surgery, were enrolled.

A diagnosis of TASS was established based on the clinical findings of a postoperative anterior chamber inflammatory response with fibrin formation in either the anterior chamber, the pupillary aperture, or just anterior to the posterior chamber intraocular lens. All subjects had no clinical evidence of posterior segment involvement (no vitreous inflammation and no acute retinal findings on a dilated fundus examination). Following a clinical diagnosis of TASS, a complete ophthalmological examination was performed including visual acuity (VA), slit-lamp examination, Goldmann applanation tonometry, and fundus examination. In addition, a baseline spectral domain OCT (SD-OCT) scan was performed.

Subjects underwent repeat biomicroscopic and SD-OCT examinations, performed on month 1 (first follow-up), month 2 (second follow-up), and a final follow-up exam performed at months 3 or 4 (third follow-up).

Spectral domain optical coherence tomography

All participants were examined using Heidelberg Spectralis SD-OCT (Heidelberg Engineering, Heidelberg, Germany), which achieves an axial resolution of $7 \mu \mathrm{m}$. The retinas of both eyes were first scanned by a horizontal high-speed raster scanning protocol of a $20^{\circ} \mathrm{X} 20^{\circ}$ quadrangular area, and central macular thickness (CMT) was obtained. Glaucoma retinal NFL protocol scan was then performed, and inferior, superior, nasal, and temporal NFL thickness measurements were recorded. In addition, in order to demonstrate choroidal thickness changes, EDI technique $[10,11]$ was used. The choroid was scanned with $20^{\circ}$ horizontal and vertical single crosssection EDI scans through the center of the fovea and by a circular EDI NFL scan protocol at the peripapilary region. To ensure high quality and noise reduction, the eye tracking system and averaging technique was employed. Each choroidal scan consisted of 100 averaged OCT frames. Images were converted to white on black grayscale to sharpen the contrast and allow a more accurate measurement. Choroidal thickness was measured by a single investigator, with an SD-OCT software electronic caliper. Choroidal thickness was defined as the vertical distance between the outer margin of the hyperreflective retinal pigment epithelium layer and the chorioscleral interface. Choroidal thickness was measured at five macular locations: subfoveal (an average of two subfoveal measurements - one from the horizontal and the other from the vertical cross-section) and $2.5 \mathrm{~mm}$ nasal, temporal, inferior, and superior to the foveal center (Fig. 1a). Similar measurements were performed in the peripapillary region in four different locations: $1.75 \mathrm{~mm}$ superior, temporal, inferior, and nasal to the optic disc center (Fig. 1b).

The follow-up acquisition mode, unique to this SD-OCT device, was used in all follow-up visits and automatically placed follow-up scans in precisely the same anatomic location as previous scans.

Two analyses of OCT measurements were performed: The first compared measurements of the study eye with measurements of the fellow eye, while the second analysis examined measurement changes in the study eye over time.

\section{Choroidal thickness measurement correction}

Previously published data demonstrated that choroidal thickness is influenced by axial length [13]. There is an inverse relationship between axial length and choroidal thickness. Therefore, choroidal thickness measurements were corrected according to the difference in axial length between the study and fellow eye. The correction coefficient used to compensate for axial length differences between the study and fellow eye was based on previously published literature [14] — for every $1 \mathrm{~mm}$ difference in axial length, choroidal thickness was reduced by $22 \mu \mathrm{m}$.

There was no need for age-related adjustments of choroidal thickness because all comparisons were performed either in the same eye or with the fellow eye of the same patient with no inter-patient comparisons.

\section{Axial length}

Axial length measurements were obtained from the patient's medical records (standard measurements performed prior to a cataract surgery). Measurements were performed using the IOLMaster (Carl Zeiss, Oberkochen, Germany) biometer. In cases of media opacities, interfering with the biometer measurements, axial length was measured using an A-scan 10$\mathrm{MHz}$ transducer with minimal corneal compression and with the use of topical anesthesia. The recorded value was the mean of six reliable measurements. 


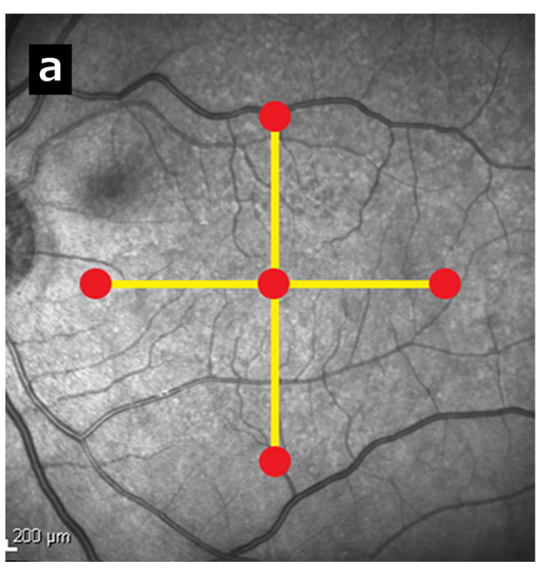

Fig. 1 a. (Patient 13) A left eye infrared map of enhanced depth imaging (EDI) optical coherence tomography (OCT) foveal measurement locations (red circles) at the foveal center and $2.5 \mathrm{~mm}$ nasal, temporal, inferior, and superior to the foveal center. The yellow lines represent the

\section{Exclusion criteria}

Exclusion criteria included cystoid macular edema of any etiology, history of endophthalmitis or other infectious process in the study eye, history of idiopathic or autoimmuneassociated uveitis in either eye, history of any posterior segment operation prior to the cataract procedure in either eye, any procedure, but for uneventful cataract surgery, patients receiving steroidal treatment (systemic or topical) prior to the cataract surgery, and media opacities preventing OCT imaging of the retina or choroid.

\section{Statistical analysis}

Data was recorded using Microsoft Excel and analyzed using SPSS version 21 (SPSS Inc., Chicago, IL, USA). Because of the small sample size, non-parametric tests were used. The Friedman test was used to compare overall difference in measurements over time (including baseline exam and the three follow-up exams) or difference between the choroidal thickness measurement locations.

Pairwise comparison of two time-point or measurement locations was analyzed by the Wilcoxon Matched-Pairs Signed-Ranks Test. All tests were two-tailed, and the threshold for statistical significance was defined as a $\mathrm{p}$ value $<0.05$.

\section{Results}

Patients and procedures

Thirteen participants were included in the study - nine women and four men. The average age was $72.8 \pm 8.7$ years (range 57-89 years). There were three left study eyes and 10 right study eyes. Two patients had bilateral epiretinal membrane

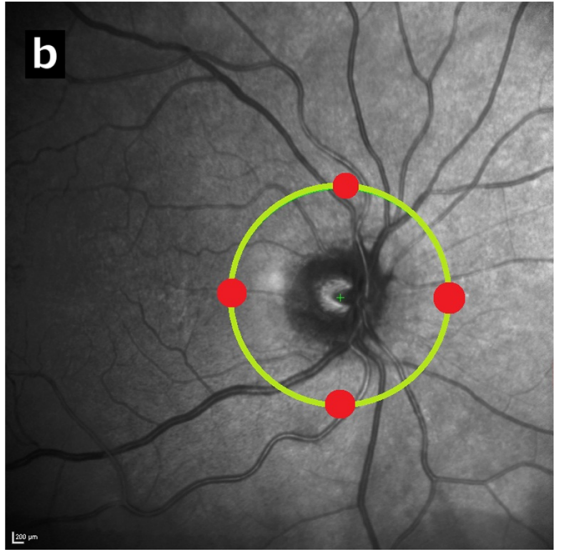

OCT cross-sections. b. (Patient 1) A right eye infrared map of EDI-OCT peripapillary measurement locations (red circles) $1.75 \mathrm{~mm}$ nasal, temporal, inferior, and superior to the optic disc center. The yellow-green circle represents the OCT cross-section

with no evidence of macular edema, and one patient had a history of branch retinal vein occlusion in his control eye with no evidence of macular edema prior to or following surgery. All patients had undergone uneventful cataract extraction surgeries.

Twelve of 13 patients had normal findings at the routine 1day postoperative exam and have been diagnosed with TASS at the routine 1-week postoperative exam (mean 8.1 days). They did not seek earlier ophthalmologic consult prior to the routine 1-week exam. One patient presented at the clinic 3 days following surgery, with TASS-related complaints and was then diagnosed. All TASS cases resolved under topical steroidal treatment (in 10 of 13 eyes, a mydriatic agent was also administered). One patient had residual posterior iris synechiae, one patient developed pseudophakic CME (diagnosed 8 weeks following TASS diagnosis), and a third patient had both residual synechiae and mild pseudophakic CME (diagnosed 10 weeks following TASS diagnosis). The remaining 10 patients had no stigmata following TASS resolution. Average follow-up times (post TASS diagnosis) for the first, second, and third follow-up exams were: day $10.5 \pm 5.6$, day $35 \pm 7.7$, and day $95.2 \pm 23.8$, accordingly. Follow-up exams included clinical evaluation and OCT exams.

\section{Macular choroidal thickness measurements}

Mean macular choroidal thickness \pm SD at the subfoveal, superior, temporal, inferior, and nasal locations was $260 \pm 84$, $248 \pm 77,219 \pm 57,197 \pm 65$, and $158 \pm 75 \mu \mathrm{m}$, respectively. All differences in macular choroidal thickness were statistically significant, except for the difference between superior and subfoveal measurements $(\mathrm{p}=0.604)$ (Table 1$)$.

Macular choroidal thickness measurements are shown in Table 2. Superior macular choroidal thickness in the study eye was significantly larger at baseline and 1st month exams (276 
Table 1 Macular Choroidal Thickness Differences (p-values shown)

\begin{tabular}{lcccl}
\hline p-Value & Subfoveal & Superior & Temporal & Inferior \\
\hline Superior & 0.604 & - & - & - \\
Temporal & 0.003 & 0.022 & - & - \\
Inferior & $<0.001$ & $<0.001$ & 0.007 & - \\
Nasal & $<0.001$ & $<0.001$ & $<0.001$ & $<0.001$ \\
\hline
\end{tabular}

\pm 74 and $276 \pm 84 \mu \mathrm{m}$, respectively) when compared with the control eye $(213 \pm 82 \mu \mathrm{m})(\mathrm{p}=0.004$ and $\mathrm{p}=0.028$, respectively). Thickness significantly decreased at the 2 nd month and $3 \mathrm{rd} / 4$ th month follow-up exams to $266 \pm 79$ and $231 \pm 40 \mu \mathrm{m}$, respectively (compared with baseline thickness in the study eye ( $p=0.018$ and $p=0.017$, respectively) (Fig. 2a).

Subfoveal and nasal macular choroidal thickness increased at baseline and 1st month exams and decreased at the 2nd and 3rd/4th month exams. These differences were not statistically significant (both for comparison with the control eye and comparison with baseline thickness in the study eye). Only one significant difference was shown in nasal choroidal thickness between study and controls eye at the 2 nd month exam (see Fig. 2b and c). No specific pattern was shown in temporal and inferior macular thickness (see Fig. 2d and e).

Peripapillary choroidal thickness measurements

Mean peripapillary choroidal thickness $\pm \mathrm{SD}$ at the superior, temporal, nasal, and inferior locations was $181 \pm 63,170 \pm 74$, $150 \pm, 60$ and $128 \pm 53 \mu \mathrm{m}$, respectively. All differences between peripapillary choroidal thickness locations were statistically significant $(p<0.001)$, except for the difference between temporal and nasal measurements $(p=0.081)$.

Table 2 Macular Choroidal Thickness Changes

\begin{tabular}{|c|c|c|c|c|c|c|}
\hline \multirow[t]{2}{*}{ Measured Location } & & & \multirow[t]{2}{*}{ Mean $(\mu \mathrm{m})$} & \multirow[t]{2}{*}{$\operatorname{STD}^{\mathrm{a}}(\mu \mathrm{m})$} & \multicolumn{2}{|l|}{ p-Value } \\
\hline & & & & & Compared with Control Eye & Compared with Study Eye Baseline \\
\hline \multirow[t]{5}{*}{ Subfoveal } & \multicolumn{2}{|c|}{ Control (Fellow) Eye } & 263.04 & 87.79 & - & - \\
\hline & \multirow[t]{4}{*}{ Study Eye } & Baseline $^{\mathrm{b}}$ & 264.83 & 84.47 & 0.638 & - \\
\hline & & Month 1 & 293.93 & 94.82 & 0.612 & 0.395 \\
\hline & & Month 2 & 262.78 & 84.51 & 0.515 & 0.058 \\
\hline & & Months 3-4 & 220.25 & 47.79 & 0.508 & 0.373 \\
\hline \multirow[t]{5}{*}{ Nasal } & \multicolumn{2}{|c|}{ Control (Fellow) Eye } & 141.92 & 62.75 & - & - \\
\hline & \multirow[t]{4}{*}{ Study Eye } & Baseline $^{\mathrm{b}}$ & 166.33 & 80.68 & 0.347 & - \\
\hline & & Month 1 & 195.14 & 87.20 & 0.128 & 0.271 \\
\hline & & Month 2 & 159.56 & 79.74 & $0.038^{*}$ & 0.735 \\
\hline & & Months 3-4 & 142.64 & 54.47 & 0.424 & 0.575 \\
\hline \multirow[t]{5}{*}{ Superior } & \multicolumn{2}{|c|}{ Control (Fellow) Eye } & 212.54 & 81.61 & - & - \\
\hline & \multirow[t]{4}{*}{ Study Eye } & Baseline $^{b}$ & 275.73 & 74.20 & $0.004 *$ & - \\
\hline & & Month 1 & 276.14 & 84.45 & $0.028 *$ & 0.398 \\
\hline & & Month 2 & 265.63 & 79.33 & 0.123 & $0.018 *$ \\
\hline & & Months 3-4 & 230.83 & 40.08 & 0.110 & $0.017 *$ \\
\hline \multirow[t]{5}{*}{ Temporal } & \multicolumn{2}{|c|}{ Control (Fellow) Eye } & 232.85 & 59.60 & - & - \\
\hline & Study Eye & Baseline $^{b}$ & 227.09 & 50.63 & 0.722 & - \\
\hline & & Month 1 & 216.86 & 53.56 & 0.735 & 0.753 \\
\hline & & Month 2 & 221.67 & 71.48 & 0.314 & 0.398 \\
\hline & & Months 3-4 & 201.00 & 52.32 & $0.022 *$ & 0.093 \\
\hline \multirow[t]{5}{*}{ Inferior } & \multicolumn{2}{|c|}{ Control (Fellow) Eye } & 186.40 & 77.23 & - & - \\
\hline & Study Eye & Baseline $^{\mathrm{b}}$ & 206.83 & 56.93 & 0.158 & - \\
\hline & & Month 1 & 193.14 & 66.50 & 0.237 & 0.612 \\
\hline & & Month 2 & 192.33 & 66.35 & 0.214 & $0.028 *$ \\
\hline & & Months 3-4 & 192.10 & 58.06 & 0.241 & 0.327 \\
\hline
\end{tabular}

a $\mathrm{STD}=$ Standard Deviation

${ }^{\mathrm{b}}$ Baseline $=$ Measurement on the day of TASS diagnosis

* Statistically Significant 
Fig. 2 Macular choroidal thickness measurements (a Superior, b Subfoveal, c Nasal, d Temporal, e Inferior). Only significant $\mathrm{p}$-values are shown. $\mathrm{p} 1$, p-value for comparison with baseline measurements in the study eye. $\mathrm{p} 2$, $p$-value for comparison with fellow eye measurements

\section{a Superior Choroidal Thickness}

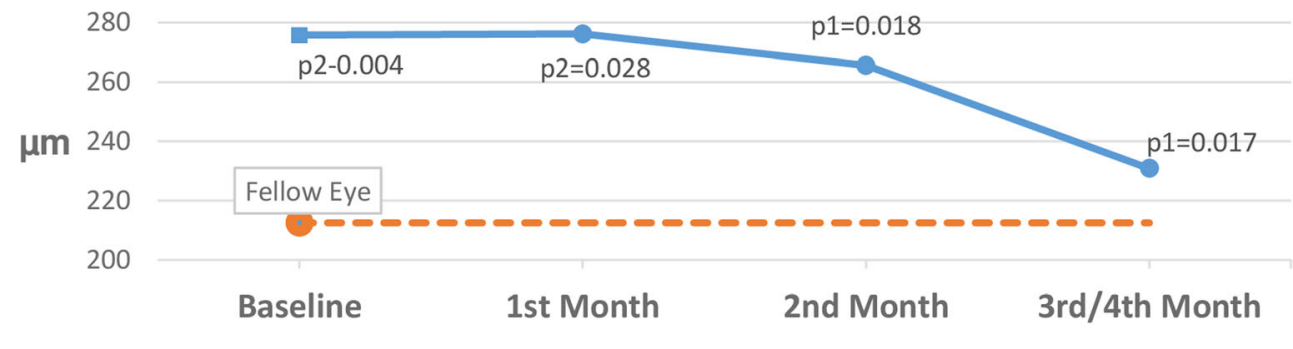

\section{b Subfoveal Choroidal Thickness}

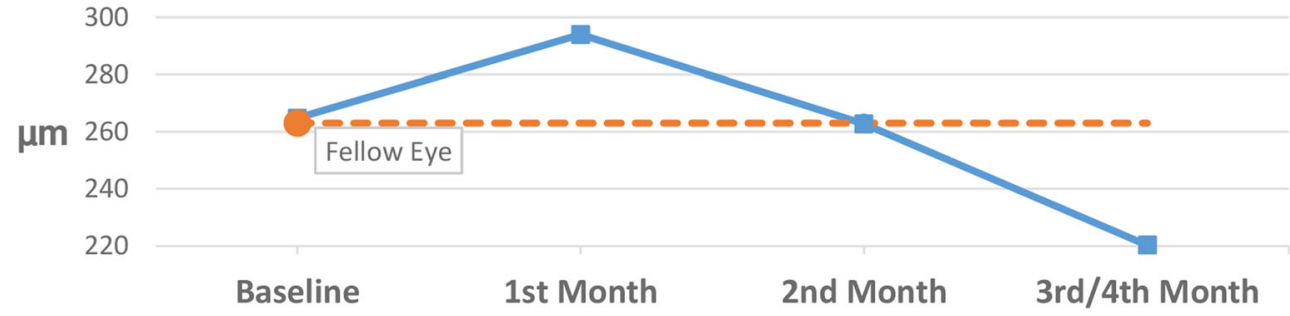

\section{c Nasal Choroidal Thickness}

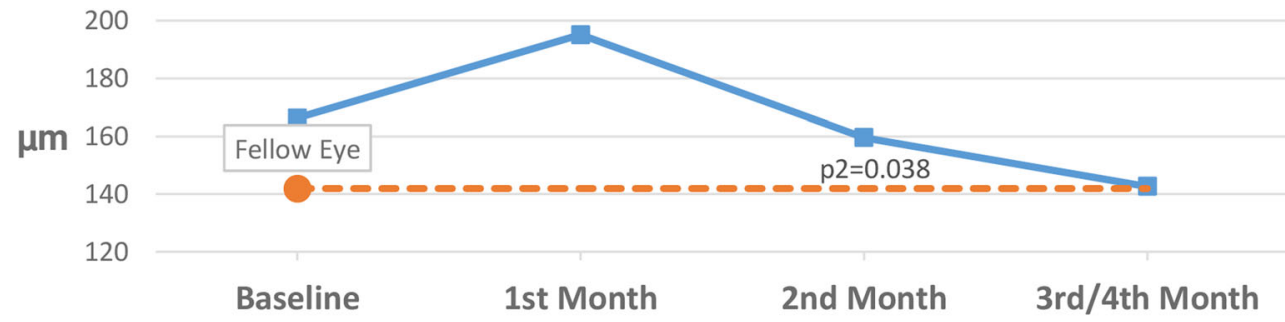

\section{d Temporal Choroidal Thickness}

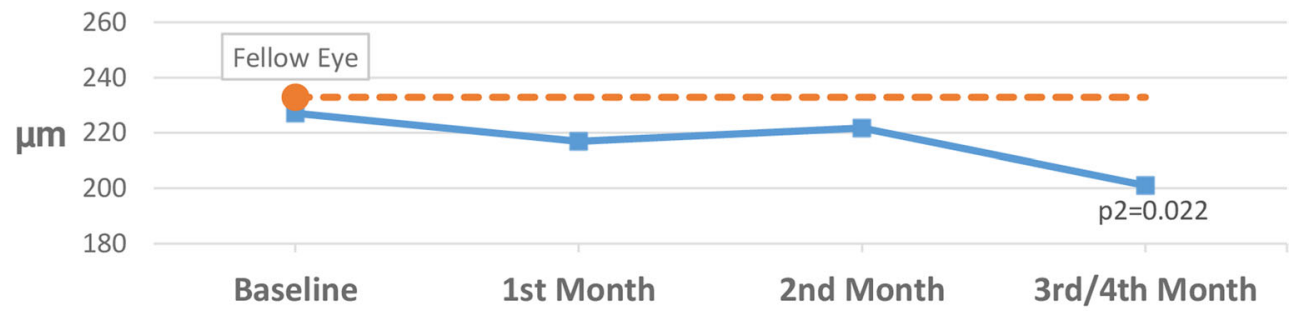

\section{e Inferior Choroidal Thickness}

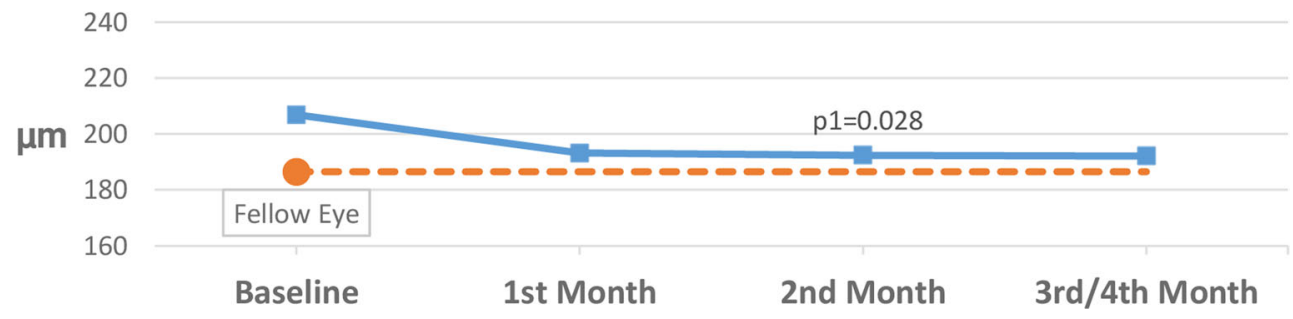

Peripapillary choroidal thickness measurements are shown in Table 3. Superior, temporal, nasal, and inferior baseline thickness in the study eye were $187 \pm 61,183 \pm 84,166 \pm 71$, and $149 \pm 61 \mu \mathrm{m}$, respectively. These were larger than control eye measurements which were $180 \pm 66,151 \pm 63,143 \pm 56$, and $116 \pm 59 \mu \mathrm{m}$, respectively. The differences were 
Table 3 Peripapillary Choroidal Thickness Changes

\begin{tabular}{|c|c|c|c|c|c|c|}
\hline \multirow[t]{2}{*}{ Measured Location } & & & \multirow[t]{2}{*}{ Mean $(\mu \mathrm{m})$} & \multirow[t]{2}{*}{$\operatorname{STD}^{\mathrm{a}}(\mu \mathrm{m})$} & \multicolumn{2}{|l|}{ p-Value } \\
\hline & & & & & Compared with Control Eye & Compared with Study Eye Baseline \\
\hline \multirow[t]{5}{*}{ Inferior } & \multicolumn{2}{|c|}{ Control (Fellow) Eye } & 116.19 & 58.66 & - & - \\
\hline & \multirow[t]{4}{*}{ Study Eye } & Baseline $^{b}$ & 149.36 & 60.74 & $0.033^{*}$ & - \\
\hline & & Month 1 & 133.50 & 54.66 & 0.133 & 0.058 \\
\hline & & Month 2 & 115.00 & 49.62 & 0.917 & 0.144 \\
\hline & & Months 3-4 & 128.80 & 55.38 & 0.285 & 0.173 \\
\hline \multirow[t]{5}{*}{ Nasal } & \multicolumn{2}{|c|}{ Control (Fellow) Eye } & 142.94 & 56.41 & - & - \\
\hline & \multirow[t]{4}{*}{ Study Eye } & Baseline $^{b}$ & 165.55 & 71.22 & $0.026^{*}$ & - \\
\hline & & Month 1 & 159.08 & 68.74 & $0.046^{*}$ & 0.833 \\
\hline & & Month 2 & 124.50 & 49.40 & 0.917 & 0.273 \\
\hline & & Months 3-4 & 151.20 & 70.69 & 0.508 & 0.066 \\
\hline \multirow[t]{5}{*}{ Superior } & \multicolumn{2}{|c|}{ Control (Fellow) Eye } & 180.03 & 66.11 & - & - \\
\hline & \multirow[t]{4}{*}{ Study Eye } & Baseline $^{b}$ & 186.82 & 60.63 & 0.790 & - \\
\hline & & Month 1 & 222.40 & 42.40 & 0.345 & 0.891 \\
\hline & & Month 2 & 158.67 & 78.07 & 0.917 & 0.080 \\
\hline & & Months 3-4 & 175.35 & 60.25 & 0.959 & 0.779 \\
\hline \multirow[t]{5}{*}{ Temporal } & \multicolumn{2}{|c|}{ Control (Fellow) Eye } & 151.65 & 62.53 & - & - \\
\hline & Study Eye & Baseline $^{b}$ & 182.60 & 84.15 & 0.386 & - \\
\hline & & Month 1 & 199.50 & 107.75 & 0.249 & 0.414 \\
\hline & & Month 2 & 179.67 & 89.20 & $0.028^{*}$ & 0.500 \\
\hline & & Months 3-4 & 143.55 & 44.06 & 0.959 & 0.726 \\
\hline
\end{tabular}

${ }^{\text {a }} \mathrm{STD}=$ Standard Deviation

${ }^{\mathrm{b}}$ Baseline $=$ Measurement on the day of TASS diagnosis

* Statistically Significant

statistically significant for the nasal $(\mathrm{p}=0.026)$ and inferior $(\mathrm{p}=0.033)$ locations. In the superior $(187 \pm 61 \mu \mathrm{m})$ and nasal $(166 \pm 71 \mu \mathrm{m})$ locations maximal thickness was at the baseline exam. In the temporal $(183 \pm 84 \mu \mathrm{m})$ and inferior $(149 \pm$ $61 \mu \mathrm{m})$ locations maximal thickness was at the 1 st month exam. These differences were not statistically significant (Table 3). Peripapillary thickness decreased thereafter (Fig. 3). Differences were not statistically significant when compared with baseline measurements in the study eye (Table 3).
Fig. 3 Peripapillary choroidal thickness measurements

\section{Peripapillary Choroidal Thickness}

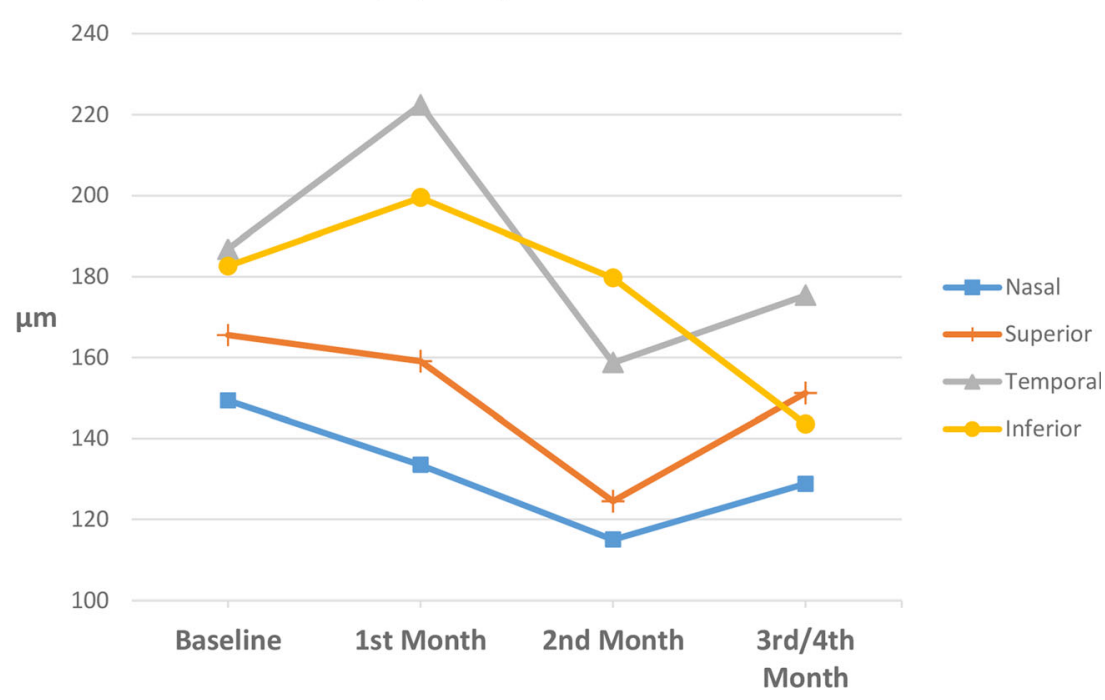


Fig. 4 Central macular thickness difference from control (fellow) eye. *Significant p-values; p-values are for comparison with fellow eye measurements

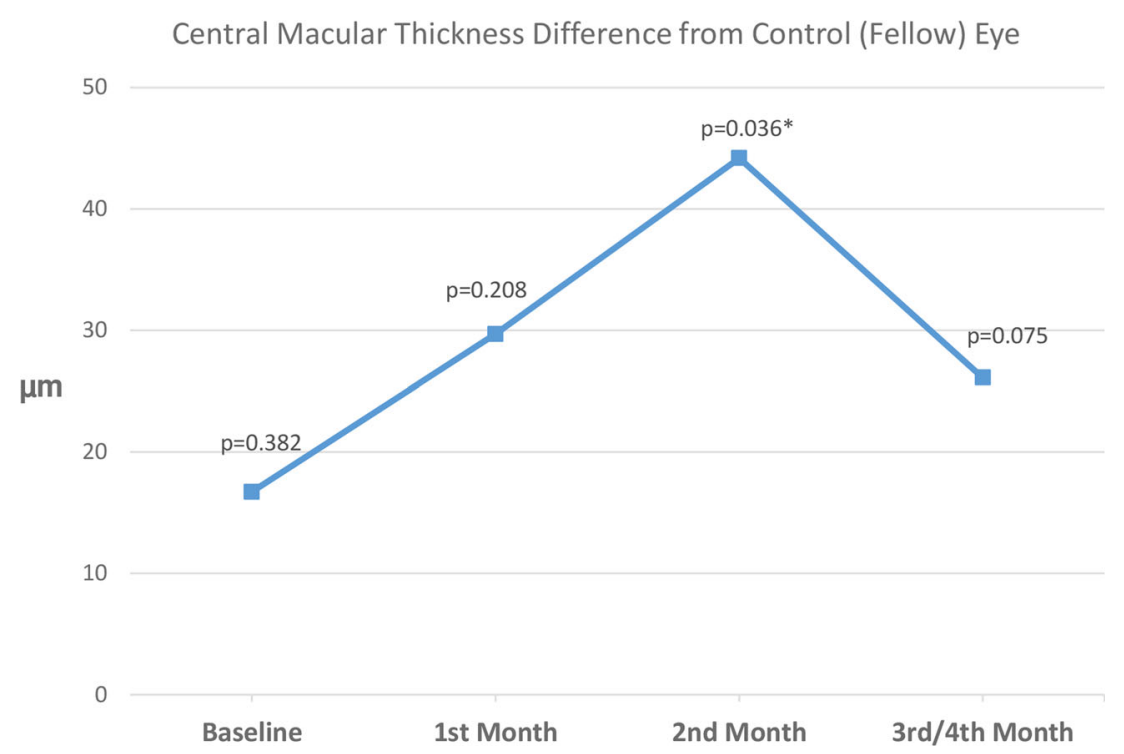

Central retinal thickness and nerve fiber layer measurements

Mean CMT at baseline was $288 \pm 48 \mu \mathrm{m}$, compared with $271 \pm$ $38 \mu \mathrm{m}$ in the control (fellow) eye. This difference of $17 \mu \mathrm{m}$ was not statistically significant ( $\mathrm{p}=0.382)$. Changes in CMT at the 1st, 2nd, and 3rd/4th month exams are shown in Fig. 4. Difference in CMT between the study and control eyes was statistically significant only at the 2 nd month exam (a difference of $44.2 \mu \mathrm{m}, \mathrm{p}=0.036$ ) (Fig. 4).

Peripapillary NFL thickness did not change significantly in any location and showed no specific pattern (Table 4).

\section{Discussion}

In this study, we evaluated quantitatively possible changes in choroidal, retinal, and nerve fiber layer thickness following an occurrence of TASS. Few studies have used EDI-OCT to evaluate inflammatory-related changes in choroidal thickness. Published studies evaluated diseases with a known chorioretinal involvement, such as Vogt-Koyanagi-Harada $[12,15]$. and Multiple Evanescent White Dot Syndrome (MEWDS) [16]. We attempted to discover subtle inflammatory changes in the choroid, resulting from an acute anterior segment inflammatory process, with no clinical evidence of posterior segment involvement.

Macular choroidal thickness in the superior, subfoveal, and nasal macula did increase and was larger than the fellow eye. This was statistically significant only for the superior macula. Peripapillary choroidal thickness was larger in the study eye at baseline compared with the control eye. This difference was statistically significant for the nasal $(\mathrm{p}=0.026)$ and inferior $(p=0.033)$ locations. There appeared to be a pattern in all peripapillary choroidal locations, showing a peak thickness at the baseline or 1st month exam with a decrease thereafter. However, these changes were not statistically significant. There are no previous publications examining choroidal thickness changes following TASS. Two recent publications examined changes in subfoveal choroidal thickness following uneventful cataract extraction and had conflicting results. The first paper found a significant increase in subfoveal choroidal thickness following cataract surgery in

Table 4 Nerve fiber layer thickness changes

\begin{tabular}{|c|c|c|c|c|}
\hline Location & & Mean $(\mu \mathrm{m})$ & $\operatorname{STD}^{\mathrm{a}}(\mu \mathrm{m})$ & p-Value* \\
\hline \multirow[t]{4}{*}{ Nasal } & Baseline $^{b}$ & 88.18 & 34.19 & - \\
\hline & Month 1 & 92.50 & 45.11 & 0.246 \\
\hline & Month 2 & 79.30 & 11.79 & 0.893 \\
\hline & Months 3-4 & 92.70 & 39.80 & 0.573 \\
\hline \multirow[t]{4}{*}{ Superior } & Baseline $^{b}$ & 124.36 & 34.40 & - \\
\hline & Month 1 & 122.42 & 38.00 & 0.753 \\
\hline & Month 2 & 114.33 & 38.72 & 0.138 \\
\hline & Months 3-4 & 123.15 & 24.39 & 0.086 \\
\hline \multirow[t]{4}{*}{ Temporal } & Baseline $^{b}$ & 71.09 & 17.68 & - \\
\hline & Month 1 & 73.92 & 22.32 & 0.596 \\
\hline & Month 2 & 70.50 & 16.02 & 0.715 \\
\hline & Months 3-4 & 67.30 & 12.07 & 0.528 \\
\hline \multirow[t]{4}{*}{ Inferior } & Baseline $^{b}$ & 123.00 & 30.11 & - \\
\hline & Month 1 & 107.17 & 22.73 & 0.600 \\
\hline & Month 2 & 118.67 & 31.77 & 0.686 \\
\hline & Months 3-4 & 128.80 & 20.16 & 0.141 \\
\hline
\end{tabular}

${ }^{\text {a }}$ STD $=$ Standard Deviation

${ }^{b}$ Baseline $=$ Measurement on the day of TASS diagnosis

* p-Values represent comparisons with Baseline measurements 
29 patients [17]. The second paper analyzed both subfoveal and peripapillary choroidal thickness measurements and found no significant change following cataract extraction [18]. Therefore, the trend found in our study may be attributed to the TASS, but evidence in the literature regarding choroidal changes following cataract extraction are inconclusive.

We also observed constant significant differences in choroidal thickness between different measurement locations. In the macular region, thickness ratio was subfoveal $>$ superior $>$ temporal $>$ inferior $>$ nasal $(260 \pm 84,248 \pm 77,219 \pm 57,197 \pm$ 65 , and $158 \pm 75 \mu \mathrm{m}$, respectively). These differences are consistent with previously published data and include nasal macular choroid is the thinnest $[10,19]$, superior macular choroid is thicker than inferior macular choroid [13, 14], subfoveal choroid is thicker than either nasal or temporal macular choroid $[11,14,19]$. Also, mean subfoveal choroidal thickness in our study $(260 \pm 84 \mu \mathrm{m})$ is similar to the value found in a recently published, large population-based study, performed on 3,233 subjects $(254 \pm 107 \mu \mathrm{m})$ [20]. In the peripapillary region, thickness ratio was superior $>$ temporal $>$ nasal $>$ inferior $(181 \pm 63,170 \pm 74,150 \pm 60$, and $128 \pm$ $53 \mu \mathrm{m}$, respectively). Previous literature shows that superior peripapillary choroid is thicker than inferior peripapillary choroid [21-23]. The correlation of our findings with previously published data strengthens the validity of our measurements and vice versa.

Measurements of retinal CMT showed an increase in retinal thickness, peaking at the 2nd month (statistically significant) and decreasing thereafter. The changes can be attributed to a transient subclinical macular inflammatory response to the cataract procedure and not to the TASS itself. Such changes following cataract surgery have been previously demonstrated in the literature [18, 24, 25]. Two patients developed CME during follow-up. In both cases, CME was not evident at the 2nd month follow-up and appeared later. Therefore, it did not seem to be a major contributor to the peak CMT change seen at month 2. No significant change in NFL thickness was observed.

In conclusion, TASS may have a transient effect on the choroid. Retinal changes observed may be related to the cataract procedure itself as previously described. We acknowledge some limitations of the study, which include a small sample size and a lack of OCT measurements prior to the occurrence of TASS. Choroidal measurements were corrected for inter-eye axial length differences, but compensating for the known diurnal variation in choroidal thickness [26, 27] was not feasible in the outpatient clinic setting.

The novel use of EDI-OCT enabled accurate imaging and quantification of choroidal changes. This study demonstrated that a significant inflammatory reaction involving the anterior uvea, such as TASS, might also have an effect on the posterior uvea. Further studies are needed to corroborate this. The continued use of EDI-OCT will help prove or refute theories of choroidal involvement in different pathologies and provide better understanding of disease processes and etiologies.

Financial support None

Conflicts of interest No conflicting relationship exists for any author

Open Access This article is distributed under the terms of the Creative Commons Attribution License which permits any use, distribution, and reproduction in any medium, provided the original author(s) and the source are credited.

\section{References}

1. Monson MC, Mamalis N, Olson RJ (1992) Toxic anterior segment inflammation following cataract surgery. J Cataract Refract Surg 18: 184-189

2. Mamalis N, Edelhauser HF, Dawson DG, Chew J, LeBoyer RM, Werner L (2006) Toxic anterior segment syndrome. J Cataract Refract Surg 32:324-333. doi:10.1016/j.jcrs.2006.01.065

3. Sengupta S, Chang DF, Gandhi R, Kenia H, Venkatesh R (2011) Incidence and long-term outcomes of toxic anterior segment syndrome at Aravind Eye Hospital. J Cataract Refract Surg 37:16731678. doi:10.1016/j.jcrs.2011.03.053

4. Cutler Peck CM, Brubaker J, Clouser S, Danford C, Edelhauser HE, Mamalis N (2010) Toxic anterior segment syndrome: common causes. J Cataract Refract Surg 36:1073-1080. doi:10.1016/j.jcrs. 2010.01.030

5. Ozcelik ND, Eltutar K, Bilgin B (2010) Toxic anterior segment syndrome after uncomplicated cataract surgery. Eur J Ophthalmol 20:106-114

6. Werner L, Sher JH, Taylor JR, Mamalis N, Nash WA, Csordas JE, Green G, Maziarz EP, Liu XM (2006) Toxic anterior segment syndrome and possible association with ointment in the anterior chamber following cataract surgery. J Cataract Refract Surg 32:227-235. doi: 10.1016/j.jcrs.2005.12.093

7. West ES, Behrens A, McDonnell PJ, Tielsch JM, Schein OD (2005) The incidence of endophthalmitis after cataract surgery among the U.S. Medicare population increased between 1994 and 2001. Ophthalmology 112:1388-1394. doi:10.1016/j.ophtha.2005.02.028

8. Wallin T, Parker J, Jin Y, Kefalopoulos G, Olson RJ (2005) Cohort study of 27 cases of endophthalmitis at a single institution. J Cataract Refract Surg 31:735-741. doi:10.1016/j.jcrs.2004.10.057

9. Wong IY, Koizumi H, Lai WW (2011) Enhanced Depth Imaging Optical Coherence Tomography. Ophthal Surg, Lasers, Imaging 42: S75-S84. doi:10.3928/15428877-20110627-07

10. Spaide RF, Koizumi H, Pozzoni MC (2008) Enhanced depth imaging spectral-domain optical coherence tomography. Am J Ophthalmol 146:496-500. doi:10.1016/j.ajo.2008.05.032

11. Margolis R, Spaide RF (2009) A pilot study of enhanced depth imaging optical coherence tomography of the choroid in normal eyes. Am J Ophthalmol 147:811-815. doi:10.1016/j.ajo.2008.12.008

12. Fong AH, Li KK, Wong D (2011) Choroidal evaluation using enhanced depth imaging spectral-domain optical coherence tomography in Vogt-Koyanagi-Harada disease. Retina (Philadelphia, Pa) 31: 502-509. doi:10.1097/IAE.0b013e3182083beb

13. Goldenberg D, Moisseiev E, Goldstein M, Loewenstein A, Barak A (2012) Enhanced depth imaging optical coherence tomography: choroidal thickness and correlations with age, refractive error, and axial 
length. Ophthalmic Surg Lasers Imaging: Off J Int Soc Imaging Eye 43:296-301. doi:10.3928/15428877-20120426-02

14. Ikuno Y, Kawaguchi K, Nouchi T, Yasuno Y (2010) Choroidal thickness in healthy Japanese subjects. Invest Ophthalmol Vis Sci 51:2173-2176. doi:10.1167/iovs. 09-4383

15. Nakai K, Gomi F, Ikuno Y, Yasuno Y, Nouchi T, Ohguro N, Nishida K (2012) Choroidal observations in Vogt-Koyanagi-Harada disease using high-penetration optical coherence tomography. Graefe's Arch Clin Exp Ophthalmol = Albrecht von Graefes Archiv fur klinische und experimentelle Ophthalmologie 250:1089-1095. doi:10.1007/ s00417-011-1910-7

16. Aoyagi R, Hayashi T, Masai A, Mitooka K, Gekka T, Kozaki K, Tsuneoka H (2012) Subfoveal choroidal thickness in multiple evanescent white dot syndrome. Clin Exp Optom : J Aust Optometrical Assoc 95:212-217. doi:10.1111/j.1444-0938.2011.00668.x

17. Noda Y, Ogawa A, Toyama T, Ueta T (2014) Long-term increase in subfoveal choroidal thickness after surgery for senile cataracts. Am J Ophthalmol. doi:10.1016/j.ajo.2014.05.016

18. Falcao MS, Goncalves NM, Freitas-Costa P, Beato JB, Rocha-Sousa A, Carneiro A, Brandao EM, Falcao-Reis FM (2014) Choroidal and macular thickness changes induced by cataract surgery. Clin Ophthalmol 8:55-60. doi:10.2147/OPTH.S53989

19. Esmaeelpour M, Povazay B, Hermann B, Hofer B, Kajic V, Kapoor K, Sheen NJ, North RV, Drexler W (2010) Threedimensional 1060-nm OCT: choroidal thickness maps in normal subjects and improved posterior segment visualization in cataract patients. Invest Ophthalmol Vis Sci 51:5260-5266. doi:10.1167/ iovs. $10-5196$

20. Wei WB, Xu L, Jonas JB, Shao L, Du KF, Wang S, Chen CX, Xu J, Wang YX, Zhou JQ, You QS (2013) Subfoveal choroidal thickness: the Beijing Eye Study. Ophthalmology 120:175-180. doi:10.1016/j. ophtha.2012.07.048

21. Vujosevic S, Martini F, Cavarzeran F, Pilotto E, Midena E (2012) Macular and peripapillary choroidal thickness in diabetic patients. Retina (Philadelphia, Pa) 32:1781-1790. doi:10.1097/IAE. 0b013e31825db73d

22. Roberts KF, Artes PH, O'Leary N, Reis AS, Sharpe GP, Hutchison DM, Chauhan BC, Nicolela MT (2012) Peripapillary choroidal thickness in healthy controls and patients with focal, diffuse, and sclerotic glaucomatous optic disc damage. Arch Ophthalmol 130: 980-986. doi:10.1001/archophthalmol.2012.371

23. Ho J, Branchini L, Regatieri C, Krishnan C, Fujimoto JG, Duker JS (2011) Analysis of normal peripapillary choroidal thickness via spectral domain optical coherence tomography. Ophthalmology 118: 2001-2007. doi:10.1016/j.ophtha.2011.02.049

24. Biro Z, Balla Z, Kovacs B (2008) Change of foveal and perifoveal thickness measured by OCT after phacoemulsification and IOL implantation. Eye 22:8-12. doi:10.1038/sj.eye.6702460

25. Gharbiya M, Cruciani F, Cuozzo G, Parisi F, Russo P, Abdolrahimzadeh S (2013) Macular thickness changes evaluated with spectral domain optical coherence tomography after uncomplicated phacoemulsification. Eye 27:605-611. doi:10.1038/eye.2013.28

26. Usui S, Ikuno Y, Akiba M, Maruko I, Sekiryu T, Nishida K, Iida T (2012) Circadian changes in subfoveal choroidal thickness and the relationship with circulatory factors in healthy subjects. Invest Ophthalmol Vis Sci 53:2300-2307. doi:10.1167/iovs. 11-8383

27. Tan CS, Ouyang Y, Ruiz H, Sadda SR (2012) Diurnal variation of choroidal thickness in normal, healthy subjects measured by spectral domain optical coherence tomography. Invest Ophthalmol Vis Sci 53:261-266. doi:10.1167/iovs. 11-8782 